\title{
Laceration repair using intranasal ketamine: a phase 2 dose escalation clinical trial
}

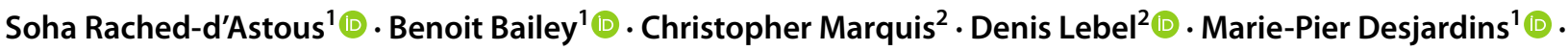 \\ Evelyne D. Trottier ${ }^{1}$
}

Received: 25 October 2021 / Accepted: 16 November 2021 / Published online: 20 December 2021

(C) The Author(s), under exclusive licence to Canadian Association of Emergency Physicians (CAEP)/ Association Canadienne de Médecine d'Urgence (ACMU) 2021

Keywords Procedural sedation $\cdot$ Analgesia $\cdot$ Intranasal $\cdot$ Pediatric $\cdot$ Ketamine

\section{Dear Editor,}

Pain and distress management options in children undergoing painful procedures which do not expose them to the additional burden of an intravenous line placement are limited. Ketamine is the most commonly used IV agent for procedural sedation [1]. Few studies, mostly for dental procedures, have evaluated its intranasal administration for procedural sedation in children, with a wide dose interval, ranging from 3 to $9 \mathrm{mg} / \mathrm{kg}$ [2]. We sought to evaluate the optimal intranasal ketamine dose for effective and safe procedural sedation in children.

We conducted a prospective dose escalation trial of IN ketamine (DosINK) for any laceration repair requiring sutures in childrenaged 1 to 12 years in the emergency department (ED). We used the most used dose-finding design, the $3+3$ escalation trial (Fig. 1) [3], for a dose range of $3 \mathrm{mg} / \mathrm{kg}$ to $9 \mathrm{mg} / \mathrm{kg}$. For each tested dose, 3 patients were enrolled and intranasal ketamine $(50 \mathrm{mg} / \mathrm{ml})$ was administered using an intranasal mucosal atomization device divided equally between the patient's nostrils. The procedure of escalation, regression and dose selection was defined a priori by the study design (Fig. 1). We used the definition of a serious adverse event and the efficacy of sedation from the PERC/PECARN consensus panel on sedation in research [4]. The primary outcome was the selected dose from the

Soha Rached-d'Astous

soharached@gmail.com

1 Department of Pediatric Emergency Medicine, CHU SainteJustine, 3175 Chemin de la Côte-Sainte-Catherine, Montreal, QC H3T 1C5, Canada

2 Department of Pharmacy, CHU Sainte-Justine, Montreal, QC, Canada escalation trial. The study, with its off-label use of ketamine, was approved by Health Canada, and by our institutional review ethics board, as well as registered at ClinicalTrials. gov (NCT03053947).

We recruited 12 patients from April 2017 to March 2018 with a median age of 2.6 (IQR $1.9,3.7$ ) years with laceration length of $2-5 \mathrm{~cm}$ and facial involvement in 10/12 (83\%) cases. Sedation was successful in $1 / 3$ patients at doses of $3 \mathrm{mg} / \mathrm{kg}$, in $1 / 3$ at $4 \mathrm{mg} / \mathrm{kg}$, in $3 / 3$ at $5 \mathrm{mg} / \mathrm{kg}$ and $3 / 3$ at $6 \mathrm{mg} / \mathrm{kg}$, all without serious adverse event. Vomiting was reported in $4 / 12(33 \%)$ patients $(0 / 3$ at $3 \mathrm{mg} / \mathrm{kg}, 1 / 3$ at $4 \mathrm{mg} /$ $\mathrm{kg}, 2 / 3$ at $5 \mathrm{mg} / \mathrm{kg}$, and $1 / 3$ at $6 \mathrm{mg} / \mathrm{kg}$ ) and nausea in $2 / 12$ (17\%) patients ( $1 / 3$ at $4 \mathrm{mg} / \mathrm{kg}$, and $1 / 3$ at $6 \mathrm{mg} / \mathrm{kg}$ ). Median time from ketamine administration to return to baseline awareness status was 45 (IQR 28, 57) minutes. When asking physicians whether they thought the medication was effective using a 5-point Likert scale, two were undecided and one strongly agreed in each of the $3 / 4 \mathrm{mg} / \mathrm{kg}$ groups, whereas two strongly agreed and one agreed in each of the $5 / 6 \mathrm{mg} / \mathrm{kg}$ groups.

The selected dose of intranasal ketamine administered for laceration repair from the escalation procedure was $6 \mathrm{mg} / \mathrm{kg}$. Our results align with the 5 and $6 \mathrm{mg} / \mathrm{kg}$ dose being used in the dental procedure literature [2]. However, the delivery system for medication administration and the medication concentration used was not clearly stated in most of the dental studies, increasing the risk of heterogeneity of the interventions and limiting comparison.

As ours is a phase 2 trial, further testing needs to be done before reaching the ED. Our results are encouraging, and the use intranasal ketamine could make a significant improvement to procedural sedation in children. 


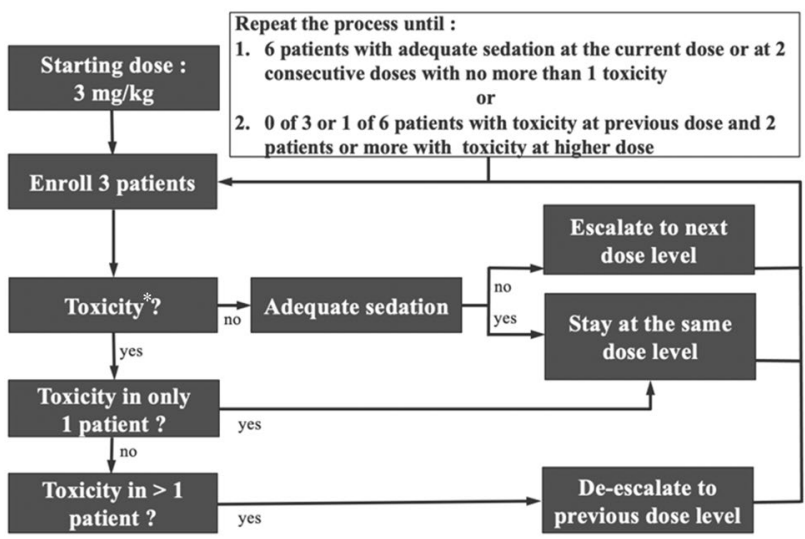

Fig. 1 Classical $3+3$ dose escalation algorithm and rules. *In our study, we use the occurrence of a serious adverse event as the definition for a toxicity

Acknowledgements Clinical Trial Registration: Pain Free Laceration Repairs Using Intra-nasal Ketamine: A Dose Escalation Clinical Trial, NCT03053947, https://clinicaltrials.gov/ct2/show/NCT03053947 .

Author contributions SRD has contributed to the study concept and design, acquisition of the data, analysis and interpretation of the data, drafting of the manuscript, and gave her final approval of the version to be published. SRD agrees to be accountable for all aspects of the work in ensuring that questions related to the accuracy or integrity of any part of the work are appropriately investigated and resolved. BB has contributed to the study concept and design, analysis and interpretation of the data, critical revision of the manuscript for important intellectual content, and gave his final approval of the version to be published. BB agrees to be accountable for all aspects of the work in ensuring that questions related to the accuracy or integrity of any part of the work are appropriately investigated and resolved. CM has contributed to the study concept and design, critical revision of the manuscript for important intellectual content, and gave his final approval of the version to be published. CM agrees to be accountable for all aspects of the work in ensuring that questions related to the accuracy or integrity of any part of the work are appropriately investigated and resolved. DL has contributed to the study concept and design, critical revision of the manuscript for important intellectual content, and gave his final approval of the version to be published. DL agrees to be accountable for all aspects of the work in ensuring that questions related to the accuracy or integrity of any part of the work are appropriately investigated and resolved. MPD has contributed to the study concept and design, critical revision of the manuscript for important intellectual content, and gave her final approval of the version to be published. MPD agrees to be accountable for all aspects of the work in ensuring that questions related to the accuracy or integrity of any part of the work are appropriately investigated and resolved. EDT has contributed to the study concept and design, acquisition of the data, analysis and interpretation of the data, drafting of the manuscript, and gave her final approval of the version to be published. EDT agrees to be accountable for all aspects of the work in ensuring that questions related to the accuracy or integrity of any part of the work are appropriately investigated and resolved.

Funding Financial support by Sainte-Justine's Youth Challenge for traumatology from CHU Sainte-Justine Foundation (Grant No. 201602).

Availability of data and material Deidentified individual participant data (including data dictionaries) will be made available, in addition to study protocols, and the informed consent form. The data will be made available upon publication to researchers who provide a methodologically sound proposal for individual participant data meta-analysis for proposal submitted up to 10 years following publication. Proposals should be submitted to soharached@gmail.com.

Code availability Not applicable.

\section{Declarations}

Conflict of interest There are no conflicts of interest or competing interests for any author.

Ethical approval Research Ethics Board of the Research Center of Sainte-Justine University Hospital, ID: 2017-1149.

\section{References}

1. Green SM, et al. Clinical practice guideline for emergency department ketamine dissociative sedation: 2011 update. Ann Emerg Med. 2011;57(5):449-61.

2. Poonai $\mathrm{N}$, et al. Intranasal ketamine for procedural sedation and analgesia in children: a systematic review. PLoS ONE. 2017;12(3):e0173253.

3. Storer BE. Design and analysis of phase I clinical trials. Biometrics. 1989;45(3):925-37.

4. Bhatt $\mathrm{M}$, et al. Consensus-based recommendations for standardizing terminology and reporting adverse events for emergency department procedural sedation and analgesia in children. Ann Emerg Med. 2009;53(4):426-435.e4. 